\title{
Endotracheal intubation-still the gold standard in out-of-hospital cardiac arrest airway management?
}

\author{
Jonathan Teng Fai Loke ${ }^{1 \wedge}$, Seth En Teoh², John J. Y. Zhang ${ }^{3} \wedge$, Yoshio Masuda ${ }^{2} \wedge$ \\ ${ }^{1}$ School of Medicine, Trinity College Dublin, Dublin, Ireland; ${ }^{2}$ Yong Loo Lin School of Medicine, National University of Singapore, Singapore, \\ Singapore; ${ }^{3}$ Ministry of Health Holdings, Singapore, Singapore \\ Correspondence to: Yoshio Masuda. Yong Loo Lin School of Medicine, National University of Singapore, 1E Kent Ridge Rd., Singapore 119228, \\ Singapore. Email: e0345860@u.nus.edu.
}

Submitted Sep 05, 2021. Accepted for publication Nov 11, 2021.

doi: 10.21037/atm-21-4668

View this article at: https://dx.doi.org/10.21037/atm-21-4668

We read with great interest the article written by Yang et al. titled "Comparing the efficacy of bag-valve mask, endotracheal intubation, and laryngeal mask airway for subjects with outof-hospital cardiac arrest: an indirect meta-analysis" (1). The authors of the study must be congratulated for their efforts in performing an indirect meta-analysis of 13 studies to compare the outcomes of bag-valve mask (BVM), laryngeal mask airway (LMA), and endotracheal intubation (ETI). Outcome measures used were return of spontaneous circulation (ROSC) and the survival rate to admission or discharge.

Approximately 300,000 persons in the United States alone experience an out-of-hospital cardiac arrest (OHCA) each year with a mortality of approximately $92 \%$ (2). This review is made even more timely by the current unprecedented global health crisis caused by the Severe Acute Respiratory Syndrome Coronavirus 2 (SARS-CoV-2). A recent systematic review identified that there has been a $120 \%$ increase in the incidence of OHCA since the pandemic, with an increase in mortality and supraglottic airway usage (3). Therefore, this article provides invaluable insights in the current conversation for OHCA management during the pandemic.

Despite the importance of effective airway management in the treatment of patients with OHCA, there is a paucity of available data on the topic, including limited high-quality Randomized Controlled Trial (RCT) data comparing the efficacy of various techniques in airway management. Randomized trials have proven to be the gold standard in analysing causal relationships as the act of randomisation and concealment of allocation eradicates inherent biases that might exist in other study designs (4). Equipoise between the various techniques have led to calls for more and larger RCTs comparing the use of BVM, LMA, and ET in the context of OHCA, to determine the most optimal device for airway management.

Of the 13 studies included by Yang et al., three studies were randomized while the rest were observational in nature. We conducted a sensitivity analysis to compare the findings of the randomized studies against the findings by Yang et al., to identify any incongruity in the findings.

Our analysis found that there were differing results for ROSC and survival to discharge when comparing BMV to LMA as compared to the values that were presented by Yang et al. On inspection of the randomized studies by Benger et al. and Fiala et al., we found no statistically significant difference when comparing BVM and LMA in the outcome of ROSC (RR $=1.00,95 \%$ CI, $0.75-1.33 ; \mathrm{I}^{2}=0 \%, \mathrm{P}=1.00$ ) (Figure 1A) $(5,6)$. Yang et al., however, found that there was a significant difference between LMA and BVM (RR $=0.84 \%$, 95\% CI, 0.57-1.24; $\left.\mathrm{I}^{2}=94.8 \%, \mathrm{P}<0.001\right)(1)$.

Further discrepancies were noted in survival to discharge when comparing BVM with LMA for the randomized studies. Our results revealed no significant difference

^ ORCID: Jonathan Teng Fai Loke, 0000-0002-0268-128X; John J. Y. Zhang, 0000-0002-8567-3807; Yoshio Masuda, 0000-0002-79315333 . 


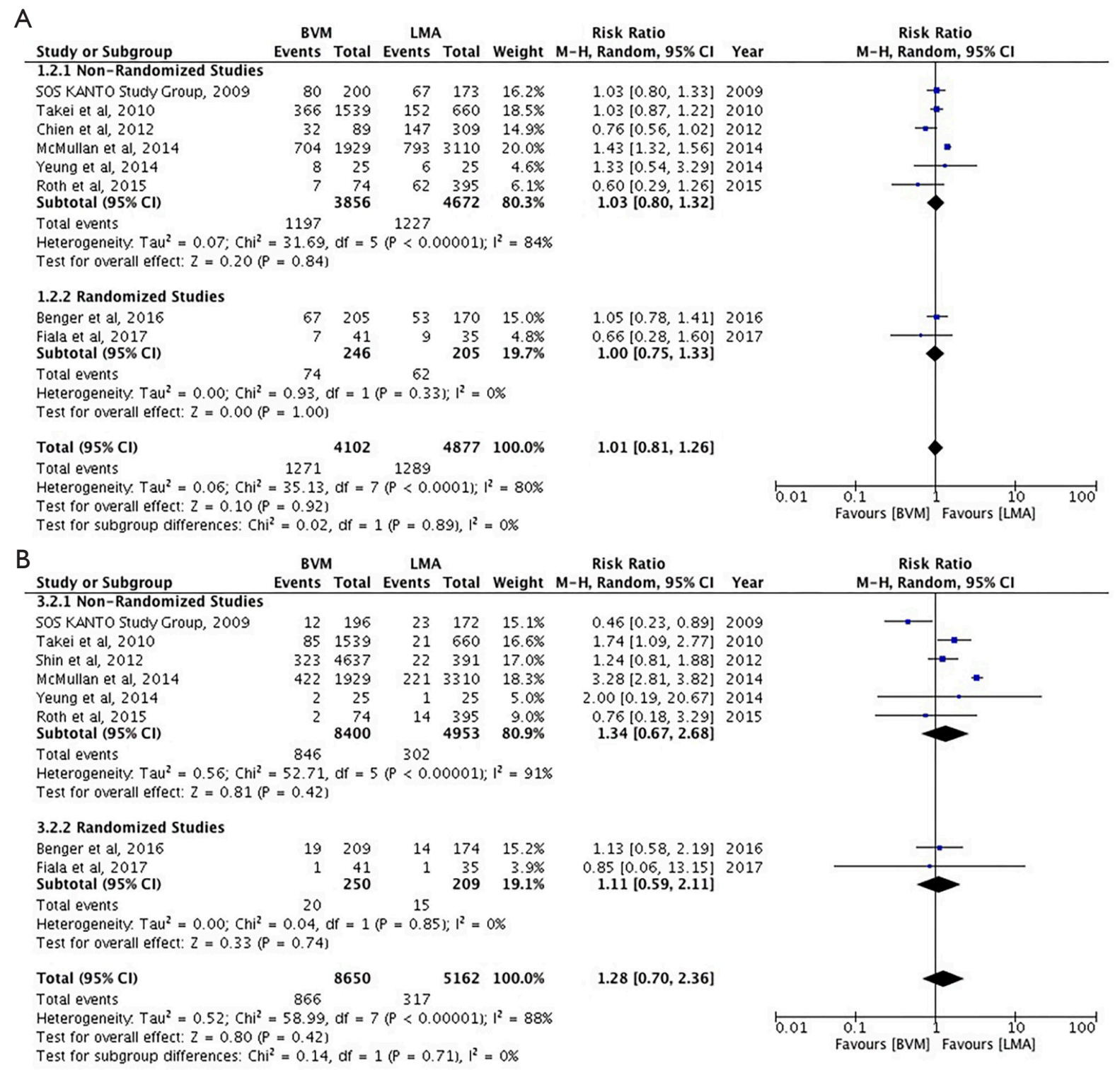

Figure 1 Forest plots of BVM and LMA for the outcome of ROSC and for the outcome of survival to discharge. (A) Forest plot comparing BVM and LMA for the outcome of ROSC; (B) Forest plot comparing BVM and LMA for the outcome of survival to discharge. BVM, bagvalve-mask; LMA, laryngeal mask airway; ROSC, return of spontaneous circulation.

between BVM and LMA (RR =1.11, 95\% CI, 0.59-2.11; $\mathrm{I}^{2}=0 \%, \mathrm{P}=0.74$ ). (Figure $1 B$ ) This is contrary to the results in the original study, whereby BVM was shown to be significantly better than LMA (RR $=0.61 ; 95 \%$ CI, 0.38 $\left.0.98 ; \mathrm{I}^{2}=79.4 \%, \mathrm{P}<0.001\right)(1)$. The study by Ono et al. was intentionally excluded from the results in Figure 1 as the use of the laryngeal tube, an extra glottic airway device, was classified under ETI instead $(7,8)$.

However, these values should be interpreted with caution as the two randomized studies listed had relatively smaller sample sizes as compared to the non-randomized counterparts. This can be attributable either to the nature of the study; as a direct consequence of randomization, or due to limitations such as number of eligible participants that fulfil the inclusion criteria, the amount of time available, and the budget allocated for the trial.

Nonetheless, there are benefits to randomized trials as it allows for the comparison of cause and effect relationships 
between interventions and outcomes (9). Randomisation also prevents any priori knowledge of group assignments and balances participant characteristics between the groups, therefore reducing any selection biases that might skew the results and allowing for any attribution of differences in outcomes to the intervention (10).

Besides the aforementioned limitations with RCTs, it is essential to acknowledge the challenges that exist especially in the context of Emergency Medicine, due to the possible ethical implications and the nature of the trial. This is particularly so with OHCA due to its unpredictable nature, making it impossible to gain the consent of patients prior to enrolment and implementation of intervention. Moreover, due to the urgency and variability in the presentation of eligible patients, randomization by patients might lead to delays in care processes and is not only impractical but potentially unethical.

Finally, there also exists a risk of residual confounding bias as numerous compounding variables such as the ventilation rate, duration of chest compression interruption for the insertion of airway adjunct, as well as other interventions that might have been performed either on-site or in-hospital, might have influences on the outcome which cannot be accounted for.

In conclusion, we acknowledge that airway management is of paramount importance in the treatment of OHCA. Although ETI has long been considered the gold standard of airway management, with the advent of alternative airway devices, there has been a recent paradigm shift regarding the most effective device for airway control. Currently, evidence on this matter remains scarce and there is a pertinent need to conduct further large scale RCTs, in order to gather more data on the efficacy of each device on the outcomes of OHCA.

\section{Acknowledgments}

Funding: None.

\section{Footnote}

Provenance and Peer Review: This article was a standard submission to the journal. The article did not undergo external peer review.

Conflicts of Interest: All authors have completed the ICMJE uniform disclosure form (available at https://dx.doi. org/10.21037/atm-21-4668). The authors have no conflicts of interest to declare.

Ethical Statement: The authors are accountable for all aspects of the work in ensuring that questions related to the accuracy or integrity of any part of the work are appropriately investigated and resolved.

Open Access Statement: This is an Open Access article distributed in accordance with the Creative Commons Attribution-NonCommercial-NoDerivs 4.0 International License (CC BY-NC-ND 4.0), which permits the noncommercial replication and distribution of the article with the strict proviso that no changes or edits are made and the original work is properly cited (including links to both the formal publication through the relevant DOI and the license). See: https://creativecommons.org/licenses/by-nc-nd/4.0/.

\section{References}

1. Yang $\mathrm{Z}$, Liang $\mathrm{H}$, Li J, et al. Comparing the efficacy of bag-valve mask, endotracheal intubation, and laryngeal mask airway for subjects with out-of-hospital cardiac arrest: an indirect meta-analysis. Ann Transl Med 2019;7:257.

2. McNally B, Robb R, Mehta M, et al. Out-of-hospital cardiac arrest surveillance --- Cardiac Arrest Registry to Enhance Survival (CARES), United States, October 1, 2005--December 31, 2010. MMWR Surveill Summ 2011;60:1-19.

3. Lim ZJ, Ponnapa Reddy M, Afroz A, et al. Incidence and outcome of out-of-hospital cardiac arrests in the COVID-19 era: A systematic review and meta-analysis. Resuscitation 2020;157:248-58.

4. Bondemark L, Ruf S. Randomized controlled trial: the gold standard or an unobtainable fallacy? Eur J Orthod 2015;37:457-61.

5. Benger J, Coates D, Davies S, et al. Randomised comparison of the effectiveness of the laryngeal mask airway supreme, i-gel and current practice in the initial airway management of out of hospital cardiac arrest: a feasibility study. Br J Anaesth 2016;116:262-8.

6. Fiala A, Lederer W, Neumayr A, et al. EMT-led laryngeal tube vs. face-mask ventilation during cardiopulmonary resuscitation - a multicenter prospective randomized trial. Scand J Trauma Resusc Emerg Med 2017;25:104.

7. Ono Y, Hayakawa M, Maekawa K, et al. Should laryngeal tubes or masks be used for out-of-hospital cardiac arrest patients? Am J Emerg Med 2015;33:1360-3. 
8. Asai T, Shingu K. The laryngeal tube. Br J Anaesth 2005;95:729-36.

9. Hariton E, Locascio JJ. Randomised controlled trials the gold standard for effectiveness research: Study design:

Cite this article as: Loke JTF, Teoh SE, Zhang JJY, Masuda Y. Endotracheal intubation-still the gold standard in out-of-hospital cardiac arrest airway management? Ann Transl Med 2021;9(23):1748. doi: 10.21037/atm-21-4668 randomised controlled trials. BJOG 2018;125:1716.

10. Suresh K. An overview of randomization techniques: An unbiased assessment of outcome in clinical research. J Hum Reprod Sci 2011;4:8-11. 